\title{
ON DISCRETE INEQUALITIES OF GRÜSS TYPE
}

\author{
SHIOW-RU HWANG AND MING-IN HO
}

Abstract. In the paper, we establish two discrete inequalities of Grüss type via inequalities of Watson-Greub-Rheiuboldt and Klamkin-Mclenaghan.

\section{Introduction}

In [6], Grüss proved the following integral inequality

$$
\left|\frac{1}{b-a} \int_{a}^{b} f(x) g(x) d x-\frac{1}{b-a} \int_{a}^{b} f(x) d x \frac{1}{b-a} \int_{a}^{b} g(x) d x\right| \leq \frac{1}{4}(\Phi-\phi)(\Gamma-\gamma)
$$

provided that $f$ and $g$ are two integrable functions on $[a, b]$ and satisfy the condition

$$
\phi \leq f(x) \leq \Phi \text { and } \gamma \leq g(x) \leq \Gamma \text { for a.e. } x \in[a, b] .
$$

The constant $\frac{1}{4}$ is the best possible in the sense that it cannot be replaced by a smaller constant.

The discrete version of (1.1) states that (see for example [2]):

If $\bar{a}=\left(a_{1}, \ldots a_{n}\right)$ and $\bar{b}=\left(b_{1}, \ldots b_{n}\right)$ are two sequences of real numbers with

$$
a \leq a_{i} \leq A<\infty \text { and } b \leq b_{i} \leq B<\infty \text { for all } i \in\{1, \ldots, n\},
$$

then

$$
\begin{aligned}
\left|C_{n}(\bar{a}, \bar{b})\right| & \leq \frac{1}{n}\left[\frac{1}{2}\right]\left(1-\frac{1}{n}\left[\frac{1}{2}\right]\right)(A-a)(B-b) \\
& =\frac{1}{n^{2}}\left[\frac{n^{2}}{4}\right](A-a)(B-b) \\
& \leq \frac{1}{4}(A-a)(B-b)
\end{aligned}
$$

where

$$
C_{n}(\bar{a}, \bar{b})=\frac{1}{n} \sum_{i=1}^{n} a_{i} b_{i}-\frac{1}{n} \sum_{i=1}^{n} a_{i} \cdot \frac{1}{n} \sum_{i=1}^{n} b_{i} .
$$

Received December 16, 2002.

2000 Mathematics Subject Classification. Primary 26D15; secondary 94A05.

Key words and phrases. discrete inequalities of Grüss Type, Watson-Greub-Rheiuboldt inequality, Klamkin-Mclenaghan inequality. 
For other new results in the domain, see the papers [1, 3-8, 9-10] and the book [8].

Recently, Dragomir and Khan [5] proved the following two discrete inequalities of Grüss type:

Theorem A. Let $\bar{a}=\left(a_{1}, \ldots a_{n}\right)$ and $\bar{b}=\left(b_{1}, \ldots b_{n}\right)$ be two $n$-tuples of positive real numbers with

$$
0<a \leq a_{i} \leq A<\infty \text { and } 0<b \leq b_{i} \leq B<\infty \text { for all } i \in\{1, \ldots, n\} .
$$

Then we have the inequality

$$
\left|C_{n}(\bar{a}, \bar{b})\right| \leq \frac{1}{4} \frac{(A-a)(B-b)}{\sqrt{A a B b}} \cdot \frac{1}{n} \sum_{i=1}^{n} a_{i} \cdot \frac{1}{n} \sum_{i=1}^{n} b_{i} .
$$

The constant $\frac{1}{4}$ is the best possible.

Theorem B. Let $\bar{a}=\left(a_{1}, \ldots a_{n}\right)$ and $\bar{b}=\left(b_{1}, \ldots b_{n}\right)$ be defined as in Theorem A. Then we have the inequality

$$
\left|C_{n}(\bar{a}, \bar{b})\right| \leq(\sqrt{A}-\sqrt{a})(\sqrt{B}-\sqrt{b}) \cdot \frac{1}{n} \sum_{i=1}^{n} a_{i} \cdot \frac{1}{n} \sum_{i=1}^{n} b_{i} .
$$

The constant $c=1$ is the best possible.

In this paper, we establish a weighted generalization of Theorem A and Theorem B, respectively.

\section{Main Results}

Theorem 1. Let $\bar{a}=\left(a_{1}, \ldots a_{n}\right)$ and $\bar{b}=\left(b_{1}, \ldots, b_{n}\right)$ be defined as in Theorem $A$, and let $\bar{p}=\left(p_{1}, \ldots, p_{n}\right)$ be an $n$-tuples of nonnegative numbers with $P_{n}=\sum_{i=1}^{n} p_{i}>0$. Then we have the inequality

$$
\left|C_{n}(\bar{p}, \bar{a}, \bar{b})\right| \leq \frac{1}{4} \frac{(A-a)(B-b)}{\sqrt{A a B b}} \cdot \frac{1}{P_{n}} \sum_{i=1}^{n} p_{i} a_{i} \cdot \frac{1}{P_{n}} \sum_{i=1}^{n} p_{i} b_{i}
$$

where

$$
C_{n}(\bar{p}, \bar{a}, \bar{b})=\frac{1}{P_{n}} \sum_{i=1}^{n} p_{i} a_{i} b_{i}-\frac{1}{P_{n}} \sum_{i=1}^{n} p_{i} a_{i} \cdot \frac{1}{P_{n}} \sum_{i=1}^{n} p_{i} b_{i}
$$

and the constant $\frac{1}{4}$ is the best possible.

Proof. By the Cauchy-Schwarz inequality, we have

$$
\left|C_{n}(\bar{p}, \bar{a}, \bar{b})\right|=\left|\frac{1}{P_{n}} \sum_{i=1}^{n} p_{i} a_{i} b_{i}-\frac{1}{P_{n}} \sum_{i=1}^{n} p_{i} a_{i} \frac{1}{P_{n}} \sum_{i=1}^{n} p_{i} b_{i}\right|
$$




$$
\begin{aligned}
& =\left|\frac{1}{2 P_{n}^{2}} \sum_{i, j=1}^{n} p_{i} p_{j}\left(a_{i}-a_{j}\right)\left(b_{i}-b_{j}\right)\right| \\
& \leq \frac{1}{2 P_{n}^{2}} \sum_{i, j=1}^{n} p_{i} p_{j}\left|\left(a_{i}-a_{j}\right)\right|\left|\left(b_{i}-b_{j}\right)\right| \\
& \leq \frac{1}{2 P_{n}^{2}}\left[\sum_{i, j=1}^{n} p_{i} p_{j}\left(a_{i}-a_{j}\right)^{2}\right]^{\frac{1}{2}}\left[\sum_{i, j=1}^{n} p_{i} p_{j}\left(b_{i}-b_{j}\right)^{2}\right]^{\frac{1}{2}} \\
& =\frac{1}{2 P_{n}^{2}}\left[2 P_{n} \sum_{i=1}^{n} p_{i} a_{i}^{2}-2\left(\sum_{i=1}^{n} p_{i} a_{i}\right)^{2}\right]^{\frac{1}{2}}\left[2 P_{n} \sum_{i=1}^{n} p_{i} b_{i}^{2}-2\left(\sum_{i=1}^{n} p_{i} b_{i}\right)^{2}\right]^{\frac{1}{2}} \\
& =\left[\frac{1}{P_{n}} \sum_{i=1}^{n} p_{i} a_{i}^{2}-\left(\frac{1}{P_{n}} \sum_{i=1}^{n} p_{i} a_{i}\right)^{2}\right]^{\frac{1}{2}}\left[\frac{1}{P_{n}} \sum_{i=1}^{n} p_{i} b_{i}^{2}-\left(\frac{1}{P_{n}} \sum_{i=1}^{n} p_{i} b_{i}\right)^{2}\right]^{\frac{1}{2}} .
\end{aligned}
$$

Using the Waston-Greub-Rheiuboldt inequality [8, p.122]

$$
\sum_{i=1}^{n} w_{i} z_{i}^{2} \cdot \sum_{i=1}^{n} w_{i} u_{i}^{2} \leq \frac{\left(M_{1} M_{2}+m_{1} m_{2}\right)^{2}}{4 m_{1} m_{2} M_{1} M_{2}} \cdot\left(\sum_{i=1}^{n} w_{i} z_{i} u_{i}\right)^{2}
$$

provided $0 \leq w_{i}<\infty, 0<m_{1} \leq z_{i} \leq M_{1}<\infty$ and $0<m_{2} \leq u_{i} \leq M_{2}<\infty$ $(i=1, \ldots, n)$, we have

$$
\left(\sum_{i=1}^{n} p_{i} a_{i}^{2}\right) \cdot P_{n} \leq \frac{(A+a)^{2}}{4 a A} \cdot\left(\sum_{i=1}^{n} p_{i} a_{i}\right)^{2}
$$

giving

$$
\frac{\left(\sum_{i=1}^{n} p_{i} a_{i}^{2}\right) \cdot P_{n}-\left(\sum_{i=1}^{n} p_{i} a_{i}\right)^{2}}{\left(\sum_{i=1}^{n} p_{i} a_{i}\right)^{2}} \leq \frac{(A+a)^{2}-4 a A}{4 a A}=\frac{(A-a)^{2}}{4 a A}
$$

thas is,

$$
\frac{1}{P_{n}} \sum_{i=1}^{n} p_{i} a_{i}^{2}-\frac{1}{P_{n}}\left(\sum_{i=1}^{n} p_{i} a_{i}\right)^{2} \leq \frac{(A-a)^{2}}{4 a A} \cdot\left(\frac{1}{P_{n}} \sum_{i=1}^{n} p_{i} a_{i}\right)^{2} .
$$

Similarly, we have

$$
\frac{1}{P_{n}} \sum_{i=1}^{n} p_{i} b_{i}^{2}-\frac{1}{P_{n}}\left(\sum_{i=1}^{n} p_{i} b_{i}\right)^{2} \leq \frac{(B-b)^{2}}{4 b B} \cdot\left(\frac{1}{P_{n}} \sum_{i=1}^{n} p_{i} b_{i}\right)^{2} .
$$

Using (2.2), (2.4) and (2.5), we otain the inequality (2.1). As note in the proof of Theorem in [5], we obtain that the constant $\frac{1}{4}$ is the best possible. This completes the proof. 
Remark 1. Choose $p_{i}=\frac{1}{n}(1, \ldots, n)$ in Theorem 1. Then the inequality (2.1) reduces to (1.4).

Theorem 2. Let $\bar{a}=\left(a_{1}, \ldots, a_{n}\right), \bar{b}=\left(b_{1}, \ldots, b_{n}\right)$ and $\bar{p}=\left(p_{1}, \ldots, p_{n}\right)$ be defined as in Theorem 1. Then we have

$$
\left|C_{n}(\bar{p}, \bar{a}, \bar{b})\right| \leq(\sqrt{A}-\sqrt{a})(\sqrt{B}-\sqrt{b})\left(\frac{1}{P_{n}} \sum_{i=1}^{n} p_{i} a_{i}\right)^{\frac{1}{2}}\left(\frac{1}{P_{n}} \sum_{i=1}^{n} p_{i} b_{i}\right)^{\frac{1}{2}} .
$$

The constant $c=1$ is the best possible.

Proof. Using the Klamkin-Mclenaghan inequality [7, p.125]

$$
\sum_{i=1}^{n} w_{i} z_{i}^{2} \cdot \sum_{i=1}^{n} w_{i} u_{i}^{2}-\left(\sum_{i=1}^{n} w_{i} z_{i} u_{i}\right)^{2} \leq(\sqrt{M}-\sqrt{m})^{2} \sum_{i=1}^{n} w_{i} z_{i} u_{i} \cdot \sum_{i=1}^{n} w_{i} u_{i}^{2},
$$

provided $0 \leq w_{i}<\infty, z_{i}>0, u_{i}>0$ and $0<m \leq \frac{z_{i}}{u_{i}} \leq M<\infty(i=1, \ldots, n)$, we have

$$
\left(\sum_{i=1}^{n} p_{i} a_{i}^{2}\right) \cdot P_{n}-\left(\sum_{i=1}^{n} p_{i} a_{i}\right)^{2} \leq(\sqrt{A}-\sqrt{a})^{2}\left(\sum_{i=1}^{n} p_{i} a_{i}\right) \cdot P_{n}
$$

that is,

$$
\frac{1}{P_{n}} \sum_{i=1}^{n} p_{i} a_{i}^{2}-\left(\frac{1}{P_{n}} \sum_{i=1}^{n} p_{i} a_{i}\right)^{2} \leq(\sqrt{A}-\sqrt{a})^{2}\left(\frac{1}{P_{n}} \sum_{i=1}^{n} p_{i} a_{i}\right) .
$$

Similarly, we have

$$
\frac{1}{P_{n}} \sum_{i=1}^{n} p_{i} b_{i}^{2}-\left(\frac{1}{P_{n}} \sum_{i=1}^{n} p_{i} b_{i}\right)^{2} \leq(\sqrt{B}-\sqrt{b})^{2}\left(\frac{1}{P_{n}} \sum_{i=1}^{n} p_{i} b_{i}\right)
$$

Using $(2.2),(2,8)$ and $(2.9)$, we obtain the inequality. As note in proof Theorem 1 in [5], we obtain that the constant $c=1$ is the best possible. This completes the proof.

Remark 2. Choose $p_{i}=\frac{1}{n}(1, \ldots, n)$ in Theorem 2. Then the inequality (2.6) reduces to $(1.5)$.

\section{References}

[1] D. Andrica and C. Badea, Grüss' inequality for positive linear functionals, Periodica Math. Hungarica 19(1988), 155-167.

[2] M. Biernacki, H. Pidek and C. Ryll-Nardzewski, Sur une inégalité entre des intégrales definies, Ann. Univ. Mariae Curie-Skolodowska A4 (1950), 1-4.

[3] S. S. Dragomir, Integral Grüss type inequality for mappings with values in Hilbert spaces and its applications, J. Korean Math. Soc. 38 (2001), 1261-1273.

[4] S. S. Dragomir, A generalization Grüss's inequality in inner product spaces and applications, J. Math. Anal. Appl. 237 (1999), 74-82. 
[5] S. S. Dragomir and L. Khan, Two discrete inequalities of Grüss type via Pólya-Szegö and Shisha results for real numbers, Tamkang J. of Math. accepted.

[6] A. M. Fink, A treatise on Grüss' inequality, Analyic and Geometric Inequalities and Applications, T. M. Rassias and H. M. Srivastava, Editors Kluwer, 1999, 93-114.

[7] G. Grüss, Über das Maximum des absoluten Betrages Von $\frac{1}{b-a} \int_{a}^{b} f(x) g(x) d x-\frac{1}{(b-a)^{2}}$ $\int_{a}^{b} f(x) d x \int_{a}^{b} g(x) d x$, Math. Z. 39 (1935), 215-226.

[8] D. S. Mitrinović, J. E. Pečarić and A. M. Fink, Classical and New Inequalities in Analysis, Kluwer Academic publishers, Dordrecht, 1993.

[9] J. E. Pečarić, On an inequality of G. Grüss, Mat. Vesnik 7 (20) (35) (1983), 59-64.

[10] J. E. Pečarić and D. Andrica, On some Grüss type inequalities, It. Sem. Func. Equat. Approx. Conv., Cluj-Napoca (1986), 221-214.

China Institue of Technology, Nankang, Taipei 11522, Taiwan.

E-mail: hsru@cc.chit.edu.tw

E-mail: mingin@cc.chit.edu.tw 